\title{
FATIGUE LIFE PREDICTION OF UMBILICALS CONSIDERING NON-LINEAR STRESS
}

\author{
Lu Qingzhen ${ }^{1}$, Lecturer \\ Yin Yuanchao', Ph. D. \\ Yang Zhixun ${ }^{2}$, Ph. D. \\ Chen Jinlong ${ }^{2}$, Ph. D. \\ Yan Jun ${ }^{2}$, Prof. \\ Yue Qianjin ${ }^{1,2}$, Prof. \\ ${ }^{1}$ School of Ocean Science and Technology, Dalian University of Tech Dalian, Liaoning, China \\ ${ }^{2}$ Department of Engineering Mechanics, Dalian University of Tech Dalian, Liaoning, China
}

\begin{abstract}
The fatigue problem induced by wave loads and floater movements of dynamic umbilicals in deepwater is studied. The prediction method of the fatigue life is investigated by considering no-linear local stress due to contact and friction between components of the umbilical. A case study of a dynamic umbilical for 1500 meters depth in South China Sea was presented. The results showed that the more accurate result of the fatigue life was calculated by considering non-linear local stress. The fatigue life by considering local stress with the no-slip assumption was conservative. An obviously longer fatigue life was obtained by considering local stress with the full-slip assumption.
\end{abstract}

Keywords: steel tube umbilical; fatigue life; contact and friction; non-linear local stress

\section{INTRODUCTION}

Steel tube umbilical is one of the key equipment in subsea production system, which supplies a communication and control link between the subsea system and surface vessels. A typical umbilical consists of various functional components for electrical power, signal transmission and hydraulic such as electric cables, optical fiber cables and steel tubes. The functional components are surrounded by amour wires and polymeric sheaths. All the components are unbonded together and can slide freely. The umblical near the vessel is usually subjected to large tension load and alternative bending load due to self-weight, currents, waves and motions of the vessel. The accumulated damage of the component is induced by the alternative load which can lead the fatigue failure of the umbilical.

The Method of the fatigue life analysis based on Miner accumulated damage theory is usually recommended by many specifications for marine engineering. However, there are still lots of uncertain factors during the analysis of the fatigue life because of the complexity of umbilical structures.

Hoffman et al.[1] and Jose et al.[2] presented a procedure of the fatigue life analysis of flexible rises and umbilicals based on Miner accumulated damage method. Song et al. [3] studied the dynamic response and the fatigue life analysis of flexible rises. The calculation method of dynamic analysis of global load had been a relatively perfect method during the fatigue life analysis of umbilicals. However, compared with 
the stress analysis of metal pipes of rigid rises which can be calculated by simple formulas, the local stress analysis was still difficult due to the nonlinear factor induced by the friction of adjacent components. Regarding the complexity and importance of the local stress of umbilical, numerous studies have been conducted in this field over last decades. Skeie et al. [4] analyzed the stress induced by tension, bending and friction, respectively. The local stress in the fatigue analysis was calculated by adding the tension stress, bending stress and friction stress. Knapp [5] established a two-dimensional finite element model of umbilical to analysis the deformation and stress. All the components in the model were modeled by macro-elements. Sævik [6,7] presented a finite element model for predicting the stress of umbilcals by curved-beam kinematics and thin-shell theory. The contact and friction between components are set by spring elements. Wang and Ekeberg [8] studied the influence of friction on the fatigue life. The results shown the fatigue life without considering friction was nearly ten time the life with considering friction. Parsinejad et al. [9] analyzed the fatigue stress and life of two different types of umbilicals by considering friction. Probyn et al. [10] established a threedimension finite element model of a steel tube umbilical by ABAQUS. Contact and Coulomb friction was defined between the adjacent components. The functional components and polymeric sheaths were modeled by solid elements and beam elements, respectively. Corre and Probyn [11] used beam elements instead of solid elements to save the calculation resources. Li and Yang [12] established a three-dimension finite element model of an umbilical with six steel tubes. The stress under different load case and the influence of friction coefficients were investigated.

Non-linear local stress due to contact and friction between components of the steel tube umbilical is presented. A numerical model is established by using Orcaflex to predict the fatigue life by considering non-linear local stress as the input parameters. A case study of a dynamic umbilical for 1500 meters depth in South China Sea was presented. The results showed that the fatigue life by considering local stress with the no-slip assumption was conservative. An obviously longer fatigue life was obtained by considering local stress with the full-slip assumption. The more accurate result of the fatigue life was calculated by considering non-linear local stress. The method presented in this paper provide a technical support for the design and application of umbilicals.

\section{ANALYSIS METHOD OF FATIGUE LIFE}

The method of the fatigue life analysis based on Miner accumulated damage theory is shown in this section. Firstly, the time-domain respond of the global load under short sea states should be calculated. Then, the time-domain respond of local stress could be analyzed under the global load. The long-term of the accumulated fatigue damage and the fatigue life are obtained by probabilistic statistics and S-N curve of the material. The method is recommended by DNV and
API standards $[13,14]$ to predict the fatigue life of marine structures. The standards also state that the predicted fatigue life of umbilical should be 10 times the service life [15].

The procedure of the fatigue analysis based on Miner accumulated damage theory is shown as below.

1. Global Analysis

Time domain analysis based on the lumped-mass method is used in the global analysis of umbilicals. The umbilical is divided to a series of segments without mass and nodes with mass. The properties of the tension, torsion and bending stiffness of the umbilical are given by the segments. The properties of the mass, gravity and buoyancy are shown on the nodes [16]. According to Longuet-Higgins equation [17], the given scatter diagrams of the irregular wave is dispersed into a serious of scatter diagrams of the regular wave. The time domain of tension and bending under each sea state is calculated, respectively. The frequency probability of the sea state in one year is $p_{i}$. The corresponding period of the sea state is $T_{z i}$. The number of the sea state in one year $n_{i}$, which can be expressed as:

$$
n_{i}=365 * 24 * 3600 * p_{i}^{*} T_{z i}
$$

\section{Local Stress Analysis}

The tension and bending load calculated in the global analysis are acting on the local structure. The local stress of the critical component can be calculated based on the Eq.(2).

$$
\sigma=K_{t} T+K_{c}\left(C_{x} \sin \theta-C_{y} \cos \theta\right)
$$

Where $K_{t}$ represents the coefficient of the tension stress, $K_{c}$ represents the coefficient of bending stress, $\theta$ is the stress of the calculated point along the circumference, $C_{x}$ represents curvature in the $x$ direction of the component, $C_{y}^{x}$ represents curvature in the $y$ direction of the component.

\section{S-N Curve of Material}

The fatigue property of the material is usually expressed by a $S-N$ curve, which is a graph of the alternating stress $\left(\Delta \sigma_{i}\right.$ ) against the logarithmic scale of cycle times to failure $\left(N_{i}\right)$

$$
\begin{aligned}
N_{i} & =10^{a}\left(\Delta \sigma_{i}\right)^{-m} \\
\log _{10}\left(N_{i}\right) & =a-m \log _{10}\left(\Delta \sigma_{i}\right)
\end{aligned}
$$

$a$ and $m$ are material constants.

\section{Fatigue Life of Umbilical}

The fatigue accumulated damage of the umbilical can be calculated based on formulas above, which can be written as:

$$
D=\sum_{i=1}^{N}\left(\frac{n_{i}}{N_{i}}\right)
$$

Where $N$ represents the number of the alternating stress, $D$ represents the fatigue accumulated damage. Fatigue failure 
occurs when $D=1$. No damage accumulates are generated when $D=0$. The fatigue life is the reciprocal of $D$, which can be expressed as:

$$
\text { Life }=\frac{1}{D}
$$

The flow of the fatigue life analysis is shown in Fig.1.

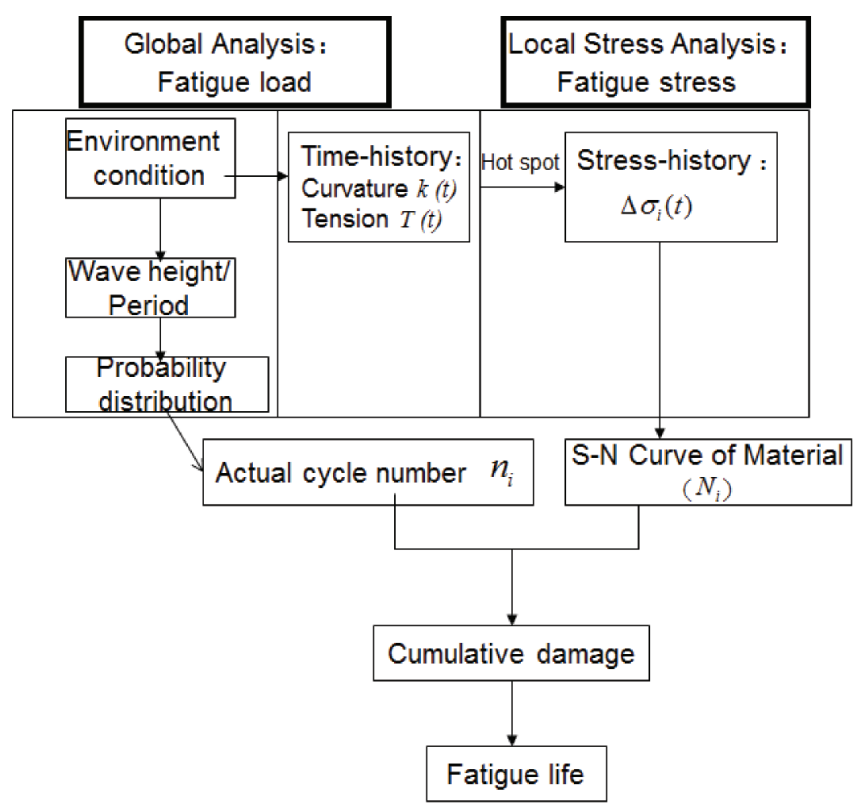

Fig. 1 Fatigue analysis flow of dynamic umbilical

The fatigue life analysis of dynamic umbilical is more difficult than steel rises. The material of steel tubes is more homogeneous compared with the material of umbilicals. The stress analysis is relatively simple because of the ring structure of the cross-section. The stress state of umbilica is complex and the calculation of the local stress is more difficult.

Non-linear local stress due to contact and friction between components of the steel tube umbilical is important for the fatigue analysis of umbilical. A more accurate fatigue life could be got based on the non-linear local stress.

\section{ANALYSIS METHOD OF LOCAL STRESS}

The structure of a typical steel tube umbilical is shown in Fig.2. The functional components and armor wires are helical wound by a special angle. The helical angle of the functional components is usually less than 10 degree. The armor wires are wound at an angle above 20 degree. The steel tube is the most critical components during the fatigue failure. The stress of the steel tube can be calculated by tension stress, bending stress and friction stress.

\section{Tension Stress}

Tension stress of each steel tube is determined by the total tension stiffness of the umbilical and the tension stiffness of the steel tube, which can be calculated by:

Where $\Delta \sigma_{T}(t)$ represents the time-domain of the tension stress, $T(t)$ represents the time-domain of the tension load, $K_{T}$ represents the tension stiffness of umbilical, $E$, $A$ denotes the elastic modulus and the cross-section area of the steel tube, respectively.

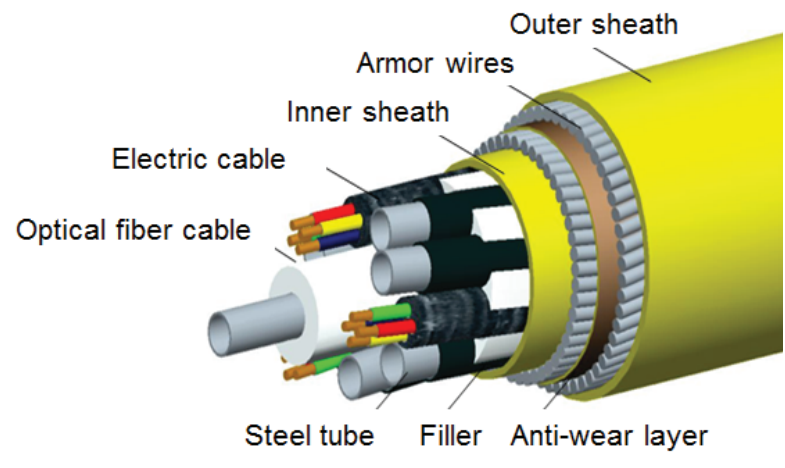

Fig.2 Compositions of a steel tube umbilical

\section{Bending Stress}

The inside conditions of umbilicals can be divided into a no-slip stage and a full-slip state during bending. The period of the no-slip stage can be increased as the increase of the tension load. The bending stress can be expressed by different stages.

\section{Bending Stress of No-slip Stage}

The steel tube is bent with the center axis of the umbilical before the component slides. The bending stress is equal to the static friction stress, which can be expressed as:

$$
\Delta \sigma_{F B}(t)=\kappa(t) E R_{L}
$$

Where $\Delta \sigma_{F B}(t)$ is the time-domain of the bending stress before slipping, $\kappa(t)$ is the time-domain of the curve, $R_{L}$ is the distance between the center of the steel tube and the umbilical.

\section{Bending Stress of Full-Slip Stage}

The bending stress caused by bending is determined by the diameter of each steel tube after the sliding of the component. The bending stress can be calculated by the formula below:

$$
\Delta \sigma_{B C}(t)=\kappa(t) E R
$$

$\Delta \sigma_{B C}(t)$ is the time-domain of the bending stress after slipping, $R$ is the diameter of the steel tube. 


\section{Friction Stress}

When the steel tube is under the full-slip stage, the static friction force turns to the dynamic friction force. The following assumptions have been made for the derivations and analysis in this paper:

1) The sliding is only occurred along the helix axis of the steel tube;

2) The end effects can be neglected;

3) The dynamic and static friction coefficients are equal.

Only one steel tube is analyzed as shown in Fig. 3. The model of the friction stress analysis can be established by the equilibrium between the axial force caused by bending and the static friction force which prevent the axial movement of the steel tube.

The sliding criterion can be established based on the equilibrium equation along the axial direction of the steel tube.

$$
\frac{d N}{d s}>f
$$

Where $N$ is the axial force, $f$ is the friction load per unit length.

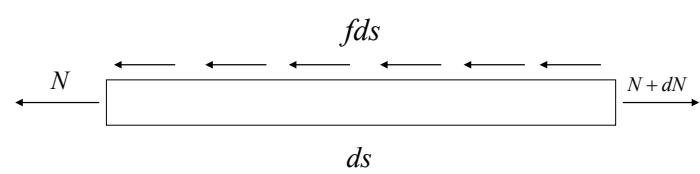

Fig.3 Force on infinitesimal helix element ds

The steel tube is assumed to be fully fixed on the cylinder. The axial load caused by bending can be expressed as:

$$
N=-E A R_{L} \cos ^{2} \alpha \sin (\varphi) \kappa
$$

Where $\alpha$ is the helical angle of the steel tube, $\varphi$ is the angle of the helical point, as shown in Fig.4. $S$ is the length of the steel tube along the helical direction. The relation between $\varphi$ and $S$ can be written as:

$$
\varphi=\frac{S}{R_{L}} \sin \alpha
$$

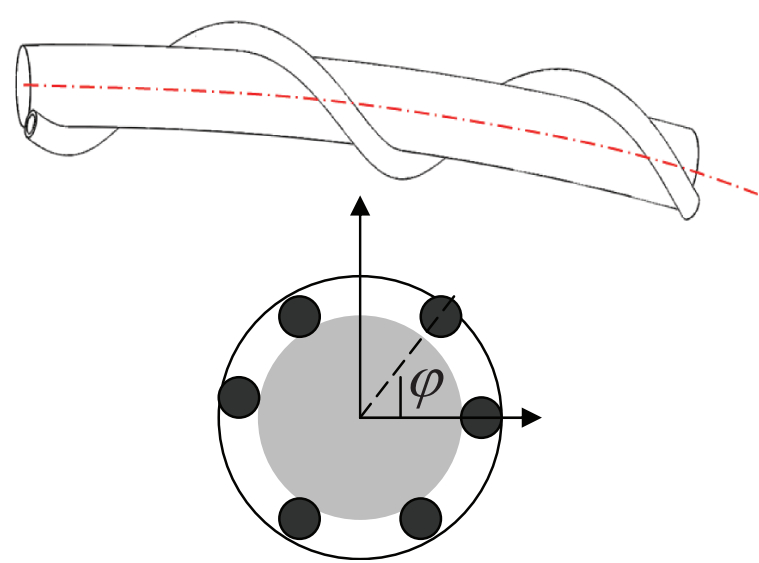

Fig.4 Helix positions for steel tube
The steel tube begin sliding when the axial force gradient $(d N / d S)$ reaches the static friction force $f$ of per unite length.:

$$
\frac{d N}{d S}=-E A \cos ^{2} \alpha \sin \alpha \cos (\varphi) \kappa_{c r}^{\min }=f
$$

The tension gradient reaches the max value when $\varphi=0$, the steel tube begins sliding at the neutral axis. The critical curvature can be established as:

$$
\kappa_{c r}^{\min }=\frac{f}{E A \cos ^{2} a \sin \alpha}
$$

The friction force $F$ equals the static friction force $N$ When the full sliding occurs at the neutral axis.

$$
\begin{gathered}
F=s f=\left(\frac{R_{L} \varphi}{\sin \alpha}\right) f=E A R_{L} \cos ^{2} a \sin (\varphi) \kappa_{c r} \\
\kappa_{c r}=\left(\frac{f}{E A \cos ^{2} a \sin \alpha}\right) \frac{\varphi}{\sin \varphi}=\kappa_{c r}^{\min } \frac{\varphi}{\sin \varphi} \\
0 \leq \varphi \leq \frac{\pi}{2}
\end{gathered}
$$

The full-slip state is assumed to be started when $\varphi=\frac{\pi}{2}$,
, max critical curvature can be expressed as:

$$
\kappa_{c r}^{\max }=\frac{\pi}{2} \kappa_{c r}^{o}
$$

The sliding friction stress can be obtained by:

$$
\sigma_{F C}=\frac{F}{A}=\frac{\pi R_{L}}{2 A \sin \alpha} f
$$

The friction force of the steel tube is affected by the friction coefficient and the length of the contact.

$$
f=\mu q_{c} L_{c}
$$

The key problem to calculate the friction force is to accurately determine the friction coefficient $\mu$ between components, the contact stress $q_{c}$ and the contact length $L_{c}$. The contact stress is analyzed by finite element model which is established by ANSYS software. The radial contact pressure induced by tension is acted on the finite element model to calculate the contact stress $q_{c}$ and the contact length $L_{c}$ of the critical steel tube. The 2-D FE model of the umbilical is shown as Fig.5. 


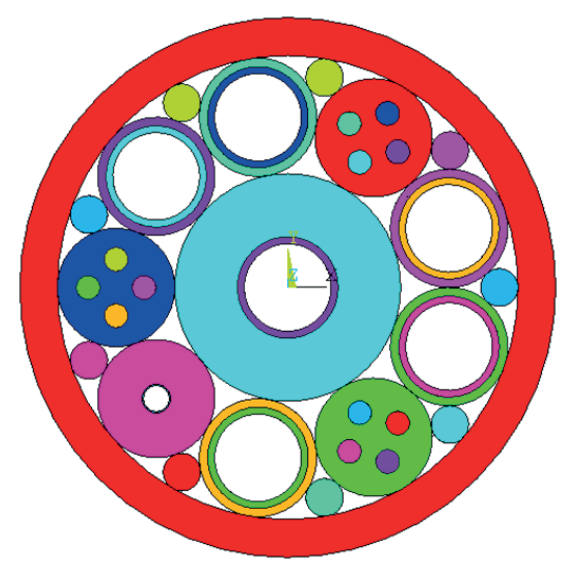

Fig.5 2-D model of the umbilical core

The transfer stage between no-slip to full slip is very short. The stress calculation of the stage is also quite difficult. So, the friction stress is assumed to be divided into two stages: no-slip stage and full-slip stage. As shown in Fig.6, the value of the no-slip stage is that of the bending stress. When the critical curvature reach to $\kappa_{c r}^{\max }$, the full-slip occurs. The value of the friction stress is that of the slip friction stress. Two straight lines is used to simulate the variation trend of the fatigue stress in this study.

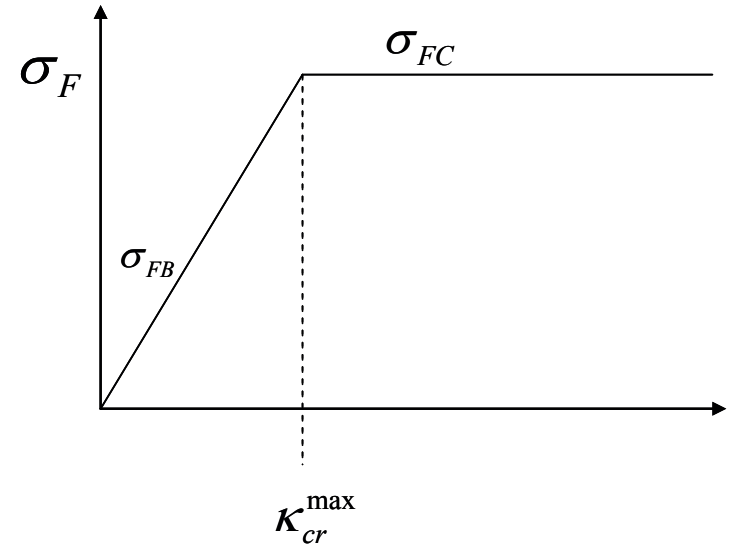

Fig.6 Definition of friction stress

\section{Local Stress}

The local stress at the no-slip stage is the sum of the tension stress and the bending stress. It can be expressed as:

$$
\Delta \sigma(t)=\Delta \sigma_{T}(t)+\Delta \sigma_{F B}(t)
$$

The local stress at the full-slip stage is the sum of the tension stress, the bending stress and the friction stress, which can be written as:

$$
\Delta \sigma(t)=\Delta \sigma_{T}(t)+\Delta \sigma_{B C}(t)+\Delta \sigma_{F C}(t)
$$

\section{EXPRERIMENT METHODS TO APPROACH FATIGUE LIFE}

As the structure of umbilical is complicated, accurate fatigue life is hard to approach by theoretical calculation or finite element method. An important way to access the fatigue life of an umbilical is to conduct a fatigue test, especially for a new designed cross section. The length in actual application of a typical dynamic umbilical is usually over a thousand meters. The sea state is also complicated. So it is impossible and useless to simulate the whole length of umbilical under the floater and wave condition in a laboratory. A feasible solution is to cut out the critical umbilical location and exert the load-time history calculated by global analysis to the test specimen by an actuator. In order to approach the fatigue life accurately, the real load case should be conducted to the test umbilical specimen by the test machine and test method, a more precise deformation of each elements should be measured.

A typical set up for a dynamic fatigue test is to simulate the real in-place condition of an umbilical while the force is tension combined with bending. The specimen was cut off at the critical location which is at the top of the umbilical. The test sample comprises the assembly of the umbilical body, end fittings, fasteners, and all devices and components simulate the umbilical top connection, and a bend stiffener/bellmouth [18]. The tension and curvature distribution at the critical area can be calculated through the global analysis of the dynamic umbilical. The tension and curvature can be applied by the force or displacement actuators at either end of the umbilical sample. Nondestructive measurement should be conducted during and after the fatigue test, which includes:

1) Tension stiffness. When the tension stiffness is declined over $20 \%$ or the rupture percent of the armor wire is over $5 \%$, the tensile armor layer is regarded as failure.

2) Inner pressure of the steel tube. The decompression should be monitored during and after the fatigue test.

3) The DC power and insulation resistance of the electric cable.

4) The signal attenuation of the optical fiber cable.

The dynamic fatigue test which simulates real working condition usually requires a large-sized test device. The length of test sample is greater than 8 meters, real end fitting and bend stiffener are also required. The whole test usually takes a long time over 3 months. The cost of the test is also very expensive. With the study of umbilical cable, a pure bending fatigue test can be conducted to test the fatigue life of umbilical for cost saving.

The load response at critical area can be obtained through global analysis. Then the local stress can be obtained by accurate analysis, which contains tension stress, bending stress and stress caused by friction. This test method can save the time and cost of a typical dynamic fatigue test. The local stress should be estimated accurately and the influence of end fittings and bend stiffness cannot be is neglected. The fatigue damage at critical area can be calculated to pure bending stress damage, which is more easy to exert by the test device. 
The fatigue property of umbilical can be tested faster by this method.

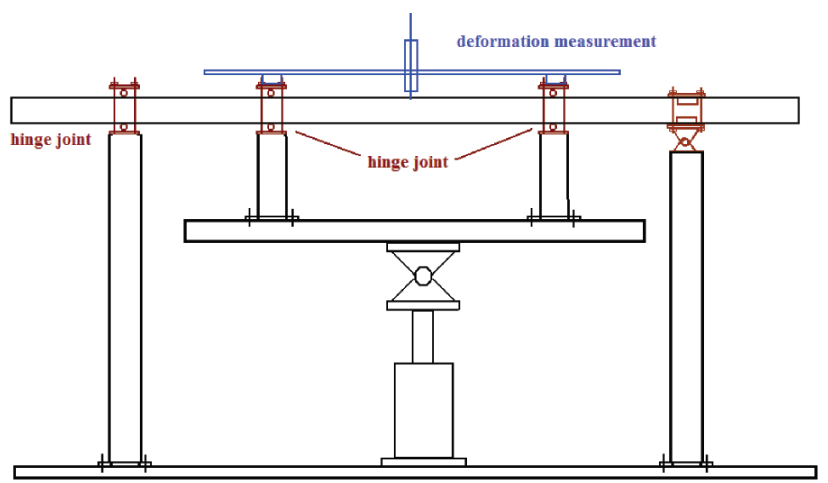

Fig.7 Testing rig of bending fatigue

The testing rig of bending fatigue is shown in Fig.7. The test specimen is placed on the holder, there were four constrained point of the test rig. The test rig should be axial symmetry during and after the fatigue test. The right constrained point on the holder is clamped to the umbilical specimen, while the specimen is free to slide at the other three points. The bending movement is exerted by a hydraulic actuator

\section{A CASE STUDY}

\section{Case Description}

A dynamic umbilical with a catenary curve of an application of $1500 \mathrm{~m}$ meters is shown in Fig.8. The seabed is plain and the solid can be considers as sandy slit. The structure of the umbilical is shown in Fig.1. The system parameters of the umbilical and the Spar platform are listed in Tab.1. The structural parameters which contains the diameter, weight, tension, torsion and bending stiffness are listed in Tab.2.

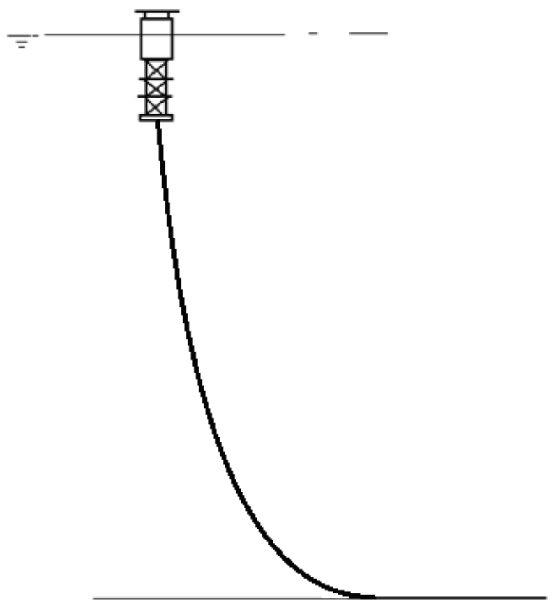

Fig. 8 A dynamic umbilical in depth of $1500 \mathrm{~m}$
Tab. 1 Parameters of umbilical system

\begin{tabular}{|l|c|c|c|}
\hline \multicolumn{1}{|c|}{ Parameters } & Value & Parameters & Value \\
\hline $\begin{array}{l}\text { Length of } \\
\text { umbilical }\end{array}$ & $1850 \mathrm{~m}$ & $\begin{array}{c}\text { Size of the soft } \\
\text { cabin }\end{array}$ & $37.2 \mathrm{~m} \times 37.2 \mathrm{~m}$ \\
\hline $\begin{array}{l}\text { Angle of top } \\
\text { point }\end{array}$ & $6^{\circ}$ & $\begin{array}{c}\text { Height of the soft } \\
\text { cabin }\end{array}$ & $6.1 \mathrm{~m}$ \\
\hline $\begin{array}{l}\text { Diameter of the } \\
\text { stiff cabin }\end{array}$ & $37.19 \mathrm{~m}$ & $\begin{array}{c}\text { Length of main } \\
\text { truss }\end{array}$ & $94.5 \mathrm{~m}$ \\
\hline $\begin{array}{l}\text { Height of the stiff } \\
\text { cabin }\end{array}$ & $80.77 \mathrm{~m}$ & Centre of gravity & 98.66 \\
\hline
\end{tabular}

Tab.2 Parameters of the umbilical

\begin{tabular}{|l|c|}
\hline \multicolumn{1}{|c|}{ Parameters } & Value \\
\hline Outer diameter $(\mathrm{m})$ & 0.1 \\
\hline Dry weight $(\mathrm{kg} / \mathrm{m})$ & 25.3 \\
\hline Wet weight $(\mathrm{kg} / \mathrm{m})$ & 15.9 \\
\hline Tension stiffness $(\mathrm{KN})$ & $3.5 e 5$ \\
\hline Torsion stiffness $\left(\mathrm{KNm}^{2}\right)$ & 35.9 \\
\hline Bending stiffness $\left(\mathrm{KNm}^{2}\right)$ & 6.6 \\
\hline
\end{tabular}

The wave scatter diagram is the probability distribution of the wave height and the period, as shown in Tab.3. The given scatter diagram of the irregular wave is dispersed into a serious of scatter diagram of the regular wave according to Longuet-Higgins equation. The coverage rate of this method can be reached $99 \%$. The cases of the regular wave used in this paper are shown in Tab. 4. The conditions of near, far and lateral position of the vessel are considered during the analysis. Each condition is set at a $33.33 \%$ probability. The proportion of a $0^{\circ}$ angle of the heat sea wave is $60 \%$, while the proportion of a $30^{\circ}$ angle is $40 \%$. The probability distribution of the wave is divide to 42 blocks of the scatter diagram of the regular wave. 252 load cases are considered in the calculation example.

A numerical model of the dynamic umbilical is established by using Orcaflex software. The joint between the umbilical and the bottom of the Spar platform is fixed with a draft of $164.6 \mathrm{~m}$. The terminal end of the umbilical is anchored to the seabed. The axial friction coefficient between the umbilical and the seabed is set as 0.25 . The overall length of the model is $1850 \mathrm{~m}$. The response amplitude operator of the movement is set at the gravity center of the platform. Different meshing is adopted in order to get the real respond of the umbilical. There is a high mesh density at the sensitive areas of the model. The whole model of the umbilical is divided into four parts. The upper area connected to the floater and the bottom area near the touchdown point are sensitive areas with a high mesh density. The length of each element of these areas is 1 meter. The static part on the seabed and the middle part of the model are divided into elements with 5 meters in length. 
Tab.3 Wave scatter diagram (Joint probability distribution of irregular wave)

\begin{tabular}{|c|c|c|c|c|c|c|c|c|c|c|}
\hline \multirow{2}{*}{\multicolumn{2}{|c|}{ Probability (\%) }} & \multicolumn{9}{|c|}{ Periods (s) } \\
\hline & & $<=3$ & $3 \sim 4$ & $4 \sim 5$ & $5 \sim 6$ & $6 \sim 7$ & $7 \sim 8$ & $8 \sim 9$ & $9 \sim 10$ & $>10$ \\
\hline \multirow{12}{*}{$\begin{array}{l}\text { Wave } \\
\text { height } \\
(\mathrm{m})\end{array}$} & $0 \sim 0.5$ & 0.29 & 1.13 & 5.35 & 8.20 & 1.47 & 0.12 & 0.01 & 0.00 & 0.00 \\
\hline & $0.5 \sim 1.0$ & 0.05 & 0.67 & 7.05 & 6.61 & 4.62 & 1.64 & 0.30 & 0.03 & 0.00 \\
\hline & $1.0 \sim 1.5$ & 0.00 & 0.02 & 1.46 & 8.34 & 5.05 & 2.06 & 0.97 & 0.37 & 0.03 \\
\hline & $1.5 \sim 2.0$ & 0.00 & 0.00 & 0.00 & 2.24 & 7.91 & 2.21 & 1.09 & 0.69 & 0.16 \\
\hline & $2.0 \sim 2.5$ & 0.00 & 0.00 & 0.00 & 0.01 & 3.72 & 4.68 & 0.86 & 0.56 & 0.33 \\
\hline & $2.5 \sim 3.0$ & 0.00 & 0.00 & 0.00 & 0.00 & 0.13 & 4.29 & 1.67 & 0.34 & 0.34 \\
\hline & $3.0 \sim 3.5$ & 0.00 & 0.00 & 0.00 & 0.00 & 0.00 & 0.91 & 3.17 & 0.38 & 0.20 \\
\hline & $3.5 \sim 4.0$ & 0.00 & 0.00 & 0.00 & 0.00 & 0.00 & 0.05 & 1.81 & 1.00 & 0.23 \\
\hline & $4.0 \sim 4.5$ & 0.00 & 0.00 & 0.00 & 0.00 & 0.00 & 0.00 & 0.33 & 1.38 & 0.30 \\
\hline & $4.5 \sim 5.0$ & 0.00 & 0.00 & 0.00 & 0.00 & 0.00 & 0.00 & 0.03 & 0.81 & 0.46 \\
\hline & $5.0 \sim 6.0$ & 0.00 & 0.00 & 0.00 & 0.00 & 0.00 & 0.00 & 0.00 & 0.17 & 0.84 \\
\hline & $>6.0$ & 0.00 & 0.00 & 0.00 & 0.00 & 0.00 & 0.00 & 0.00 & 0.02 & 0.86 \\
\hline
\end{tabular}

Tab.4 Regular wave cases for fatigue calculation of umbilical

\begin{tabular}{|c|c|c|c|c|c|c|c|c|c|}
\hline $\begin{array}{c}\text { Wave } \\
\text { height }\end{array}$ & \multicolumn{9}{|c|}{ Probability } \\
\hline 13 & & & & 12.4 & 14.4 & & & & \\
\hline 11 & & & 1.9 & 77.1 & 80.6 & 2.4 & & & \\
\hline 9 & & & 33.5 & 422.9 & 378.9 & 23.8 & & & \\
\hline 7 & & & 522.1 & 2095.9 & 1450.6 & 159.6 & 7.36 & & \\
\hline 5 & & 137.2 & 7660.4 & 10058.3 & 5165.8 & 820.1 & 80.28 & 27.1 & 4.2 \\
\hline 3 & 183.9 & 24073.8 & 85651 & 42299.1 & 18816.5 & 4176 & 675.8 & 348.7 & 86.5 \\
\hline 1 & 383617 & 531684 & 354077 & 106386 & 53435.6 & 18850 & 4931.0 & 3681.4 & 1326 \\
\hline Period & 3 & 5 & 7 & 10 & 12 & 18 & 25 & 30 & 50 \\
\hline
\end{tabular}

2. Analysis of Local Stress

The steel tube of the innermost layer is squeezed by the armor wires and adjacent components. The contact pressure is analyzed by finite element method of the model which is shown in Fig. 5. The results of the contact stress is shown in Fig.9. The biggest contact stress is occurred at the points where the outer steel tubes couple with the inner steel tube. The value of the contact stress are extracted from the crossover elements. The result shows that the maximum contact stress is at the connection area between the outer tube and the center tube. The value of the contact stress of all steel tube elements along the circle is shown in Fig. 10.

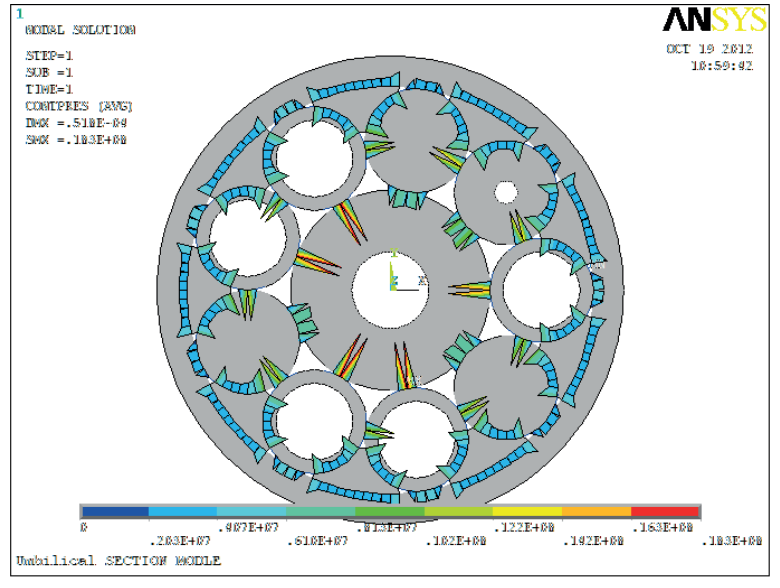

Fig. 9 Result of contact stress 


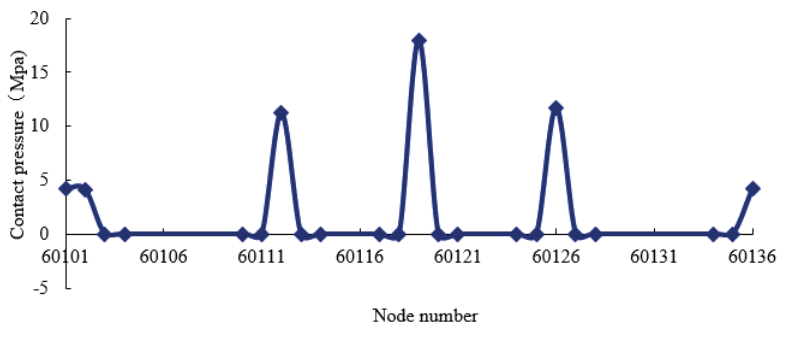

Fig.10 Contact stress values of the nodes along the tube

The critical curvature where the components begin to slide can be calculated by Eq. (17). The value of the sliding friction stress of each unit length can be obtained by Eqs. (18), (19). The total stress as the change of curvature can be got by Eqs. (20), (21). In addition, the other two assumptions of the stress condition are also considered. In the no-slip assumption, the stress is equal to the static frictional stress which can be calculated by Eq. (20). In the full- slip assumption, the stress is obtained based on Eq. (21). The results of the total stress under three conditions are shown in Fig. 11.

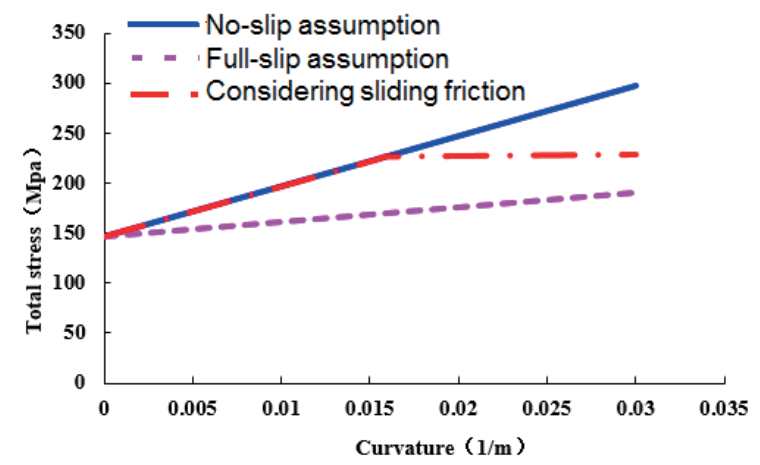

Fig. 11 Results of the total stress

\section{Prediction of Fatigue Life}

The load case of fatigue at the upper dangerous point are considered. When the stress considering the no-slip assumption are inputted, the calculated fatigue life is 1850 years. The service life is 185 years by considering a safety factor of 10 times. The fatigue life is 250 years by considering the non-linear stress as input. It is 35\% larger than the life calculated under the no-slip assumption. When the stress considering the full-slip are inputted, the calculated life is 3116 years, which is far larger than the other conditions.

\section{TEST VERIFICATION}

Dynamic fatigue test can be conducted by a pure bending fatigue device to qualify an umbilical for its service life. The fatigue test stated in this paper is to qualify the 250 years' service life of a dynamic umbilical.

The loading procedure of the fatigue test should be given by the global analysis. The relationship of alternating stress and the probability of the actual number can be accessed by post processing of umbilical fatigue life analysis. Each square of the rectangle represents the occurrence times probability of a range of alternating stress as shown in Fig. 7. The total times in Fig 7 is $91 \%$. The relationship between alternating stress $\Delta \sigma$ and pure bending curvature $\kappa$ can be established as:

$$
\Delta \sigma=2 \times E \times R \times \kappa
$$

The alternating stress in Fig. 12 can be equivalent to pure bending curvature. The fatigue amplitude should be accelerated for determine the loading of fatigue test. The curvature of $0.03,0.04,0.05$ and 0.06 were chosen to conduct the fatigue test. The probability of occurrence times of each curvature is $36 \%, 24 \%, 16 \%$ and $15 \%$. The curvature and damage times of alternating stress based on S-N curve is shown in Tab. 5 .

Tab.5 Curvature and damage times of alternating stress

\begin{tabular}{ccc}
\hline type & $\begin{array}{c}\text { curvature } \\
(\mathrm{rad} / \mathrm{m})\end{array}$ & $\begin{array}{c}\text { damage times of } \\
\text { alternating stress }\end{array}$ \\
\hline 1 & 0.03 & 12501524.45 \\
2 & 0.04 & 4567487.80 \\
3 & 0.05 & 2091666.06 \\
4 & 0.06 & 1104989.08 \\
\hline
\end{tabular}

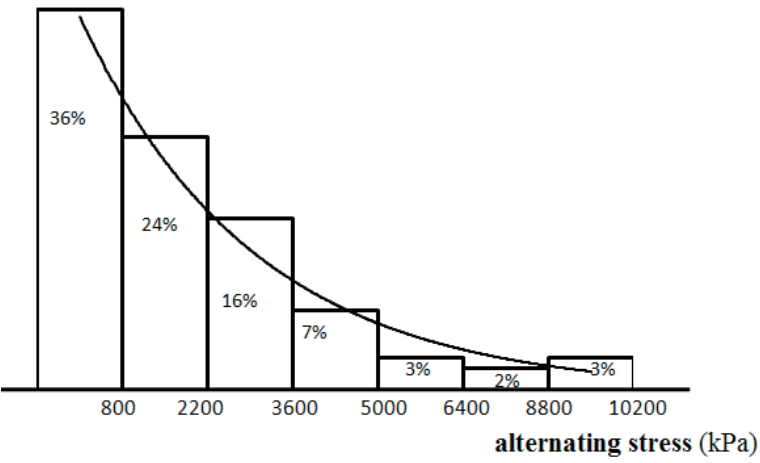

Fig. 12 Alternating stress-times probability

Where $f_{i}$ is the loading frequency (which is $0.5 \mathrm{~Hz}$ in this test) and $h_{i}$ is the loading time(hours).

The relationship between accumulated damage $D_{i}$ and loading times $n_{i}$ and total number $N_{i}$ can be accessed by Miner accumulated damage theory, which can be established as:

$$
D_{i}=\frac{n_{i}}{N_{i}}
$$

The fatigue life of the umbilical is 1850 years. In order to guarantee the service life of 250 years, the load case times 
should get the fatigue damage to $D_{i}=250 / 1850=0.176$. The load case of fatigue test is shown in Table 6.

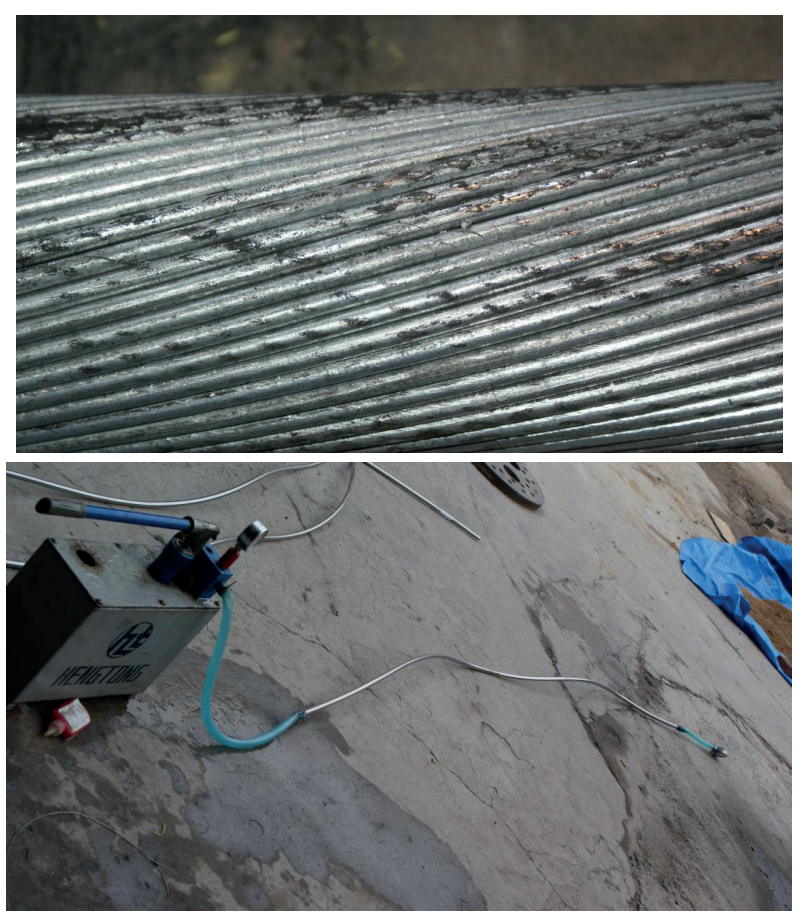

Fig. 13 Broken-out section view of umbilical after test

In order to check the fatigue damage of the body and each element of the umbilical, a dissection was conducted to the umbilical after the test which is shown in Fig. 13. There was wear between the armor wires and no damage happens. A hydrostatic pressure test was conducted to the steel tube with a $2 \mathrm{Mpa}$ inner pressure. No decompression or leakage occurs during the test, which indicates there was no damage of the steel tubes.

\section{CONCLUSION}

The fatigue life of umbilicals is investigated in this paper. A prediction model of the fatigue life is presented. Non-linear stress due to contact and friction between components of umbilicals is considered during the local analysis in this method. A numerical model was established by Orcaflex software based on Miner accumulated damage theory. A case study of a dynamic umbilical for 1500 meters depth in South
China Sea was presented. The fatigue life of an umbilical is verified by a pure bending fatigue test. The results show that:

1) The fatigue life of the umbilical by considering local stress with the no-slip assumption was conservative. The difference is $35 \%$, which may cause unnecessary waste for engineer.

2) An obviously longer fatigue life was obtained by considering local stress with the full-slip assumption, which has a certain risk.

The more accurate result of the fatigue life was calculated by considering the non-linear local stress as the inputs. The conclusion of this paper provides a technical support for design of and application dynamic umbilical.

\section{ACKNOWLEDGMENTS}

Financial support for this research was provided by the program of NSFC (51509034) and the National High Technology Research and Development Program of China (2014AA09A224). Their supports are gratefully appreciated.

\section{BIBLIOGRAPHY}

1. Hoffman J: Dupont W, Reynolds B. A fatigue-life prediction model for metallic tube umbilicals. Proceeding of Offshore Technology Conference, Houston, 2001.

2. de Sousa J R M, de Sousa F J M, de Siqueira M Q, et al: A theoretical approach to predict the fatigue life of flexible pipes. Journal of Applied Mathematics, (2012), 2012, p.1-29.

3. Song L J, Fu S X, Chen X Q: Deepwater umbilical overall response characteristic comparison research. Journal of Vibration and Shock (China), 33(1), 2014, p.119-124.

4. Skeie G, Sodahl N, Steinkjer O: Efficient fatigue analysis of helix elements in umbilicals and flexible risers: Theory and applications. Journal of Applied Mathematics, (2012), 2012, p.1-22.

5. Knapp R H: Structural analysis of composite umbilical cables. International Society of Offshore and Polar Engineers, Lisbon, 2007, p.3487-3491.

Tab. 6 Load case of fatigue test

\begin{tabular}{ccccccc}
\hline type & $\begin{array}{c}\text { curvature } \\
(\mathrm{rad} / \mathrm{m})\end{array}$ & $\begin{array}{c}\text { frequency } \\
(\mathrm{Hz})\end{array}$ & $\begin{array}{c}\text { time } \\
\text { (hours) }\end{array}$ & test times & $\begin{array}{c}\text { damage times of } \\
\text { alternating stress }\end{array}$ & $\begin{array}{c}\text { fatigue } \\
\text { damage }\end{array}$ \\
\hline 1 & 0.03 & 0.5 & 96 & 172800 & 12501524 & 0.014 \\
2 & 0.04 & 0.5 & 75 & 135000 & 4567487 & 0.030 \\
3 & 0.05 & 0.5 & 52 & 93600 & 2091666 & 0.045 \\
4 & 0.06 & 0.5 & 50 & 90000 & 1104989 & 0.081 \\
\hline
\end{tabular}


6. Sævik S: Theoretical and experimental studies of stresses in flexible pipes. Computers \& Structures, 89(23), 2011, p. 2273-2291.

7. Sævik S: Comparision between theoretical and experiment flexible pipe bending stresses. International Conference on Ocean, Offshore Mechanics and Artic Engineering, Shanghai, June 6 to11, 2010.

8. Wang $\mathrm{H} \mathrm{H}$, Ekeberg K I: Internal friction effects on fatigue life of steel tube umbilical. The 24th International Ocean and Polar Engineering Conference. Busan, June 15 to 20, 2014.

9. Parsinejad F, Kassner C, Kurtz M, et al: Friction, contact pressure, and nonlinear behavior of steel tubes in subsea umbilicals. Journal of energy resources technology, 137(3), 2015, p.032906-032913.

10. Probye I, Dobsom A: Michael Martinez. Advances in 3D FEA techniques for metallic tube umbilicals. International Society of Offshore and Polar Engineers, Lisbon, 2007, p.848-854.

11. Le Corre V, Probyn I: Validation of a 3-Dimensionalfinite element analysis model of deep water steel tube umbilical in combined tension and cyclic bending. The 28th International Conference on Ocean, Offshore and Arctic Engineering. Hawaii, May 31 to June 11, 2009, p.77-86.

12. Li Q Q, Yang H Z: Static Analysis of the Tubes Interaction in Deep-sea Umbilicals. Journal of China Offshore Platform (China), (6), 2011 p.28-32.

13. API RP 2A: Recommended practice for planning, design, and constructing fixed offshore structures.1991.

14. DNV. Rules for the design, construction, and inspection of fixed offshore structures.

15. ISO 13628-5: Petroleum and natural gas industries-Design and operation of subsea production systems-Part 5: Subsea Umbilicals.2009.

16. Orcina Ltd: Orcaflex Manual,Version 9.4a,2010.

17. Longuet-Higgins M S: On the joint distribution of wave periods and amplitudes in a random wave field. Proceedings Royal Society London, Mathematical and Physical Sciences.389, 1983, p.241-258.

18. Api, R. P. (2008). 17B. Recommended practice for flexible pipe, 4.

\section{CONTACT WITH THE AUTHOR}

Lu Qingzhen

e-mail:luqingzhen@dlut.edu.cn

School of Ocean Science and Technology

Dalian University of Tech

Dalian, Liaoning

124221 CHINA 